\title{
ON THE IMBEDDING PROBLEM OF NORMAL ALGEBRAIC NUMBER FIELDS
}

\author{
EIZI INABA
}

Let $G$ and $H$ be finite groups. If a group $\bar{G}$ has an invariant subgroup $\bar{H}$, which is isomorphic with $H$, such that the factor group $\bar{G} / \bar{H}$ is isomorphic with $G$. then we say that $\bar{G}$ is an extension of $H$ by $G$. Now let $G$ be the Galois group of a normal extension $K$ over an algebraic number field $k$ of finite degree. The imbedding problem concerns us with the question, under what conditions $K$ can be imbedded in a normal extension $L$ over $k$ such that the Galois group of $L$ over $k$ is isomorphic with $\bar{G}$ and $K$ corresponds to $\bar{H}$. Brauer connected this problem with the structure of algebras over $k$, whose splitting fields are isomorphic with $K$ 。 Following his idea, Richter investigated its local aspect using the norm theorem in the class field theory. Considering the case, where $G$ is a $p$-group and the order of $H^{r}$ is $p$, Scholz, Reichardt, and Tannaka succeeded to construct a normal extension over $k$, whose Galois group is a given $p$-group with $p \neq 2$. Scholz also solved the case, where $G$ and $H$ are both abelian. In spite of the efforts of these mathematicians the general case remains in a situation very difficult to approach. In the present paper we shall investigate the case, where $G$ is arbitrary and $H$ abelian of type $(p, \ldots, p)$ for a prime number $p$. In view of the fact, that every solvable group has a chief series $\left\{G_{i}\right\}$ such that the factor groups $G_{i} / G_{i+1}$ are abelian of type $(p, \ldots, p)$, the following investigation shall be available for the construction of normal extensions with solvable groups.

In the following we identify $\bar{H}$ with $H$. Let $g_{s} \in \bar{G}$ be a representative of the coset, which corresponds to $s \in G$. We denote with $s h$ the element $g_{s} h g_{s}^{-1}$ $\in H$, which is uniquely determined for $s \in G$ and $h \in H$ irrespective of the choice of $g_{s}$ from the coset. $H$ becomes a $G$-module by this operation and yields a representation $A$ of $G$. If the rank of $H$ is $n$, then every element in $H$ can be regarded as an $n$-dimensional vector, whose components are integers mod. $p$. If it corresponds a matrix $\Lambda(s)$ for $s \in G$ in the representation $\Lambda$, then $s h=\Lambda(s) h$. From $g_{s} g_{t}=A(s, t) g_{s t}$ with $A(s, t) \in H$ it follows

$$
A(s, t)+A(s t, u)=A(s, t u)+\Lambda(s) A(t, u),
$$

where $A(s, t)$ is called the factor set of the extension $\bar{G}$ of $H$ by $G$. If we take $g_{s}^{\prime}=B(s) g_{s}$ with $B(s) \in H$ in place of $g_{s}$, then we have a factor set $A^{\prime}(s, t)$, which

Received September 19, 1951. 
is equivalent to $A(s, t)$, and

$$
A^{\prime}(s, t)=A(s, t)+B(s)-B(s t)+A(s) B(t) .
$$

The transformation of the basis of $H$ gives rise to a representation $D A D^{-1}$, which is equivalent with $\Lambda$. In this case we obtain the factor set $D A(s, t)$ in place of $A(s, t)$. It is well known that the extension of $H$ by $G$ is uniquely determined up to isomorphism by the class of representations and the class of factor sets.

Now let $S$ be a subgroup of $G$. If there exists $B(\sigma) \in H$ for every $\sigma \in S$ such that

$$
A(\sigma, \tau)=B(\sigma)-B(\sigma \tau)+A(\sigma) B(\tau)
$$

for every $\sigma, \tau \in S$, then we say that $A(s, t)$ splits relative to $S$. In this case $A(s, t)$ is equivalent to a factor set $A^{\prime}(s, t)$ such that $A^{\prime}(\sigma, \tau)=0$ for every $\sigma$, $\tau \in S$.

LeMmA. $v$ being any fixed element in $G, A(v) A\left(v^{-1} s v, v^{-1} t v\right)$ is a factor set, which is equivalent to $A(s, t)$.

This lemma can be easily verified, if we choose $g_{s}^{\prime}=g_{v} g_{v^{-1} s v} g_{v}^{-1}$ as the representative of the coset $g_{s} H$ in place of $g_{s}$. From this lemma we have readily

THEOREM 1. If $A(s, t)$ splits relative to $S$, then it splits also relative to any conjugate subgroup $v^{-1} \mathrm{~S} v$ of $S$.

Theorem 2. Let $S$ be a p-Sylow subgroup of $G$. If $A(s, t)$ splits relative to $S$, then it splits relative to $G$. Two factor sets are equivalent to each other, if their difference splits relative to $S$.

Proof. Let $t_{i} S, i=1, \ldots, r$, be all left cosets of $S$ in $G$. We can assume that $A(\sigma, \tau)=0$ for every $\sigma, \tau \in S$ and $A\left(t_{i}, \sigma\right)=0, i=1, \ldots, r$, for every $\sigma \in S$, if we put $g_{t \sigma}=g_{t_{i}} g_{\sigma}$. Since we have from (1) $A(s, \sigma)=0$ for every $\sigma \in S$ and $s \in G$, it follows $A(s, t)=A(s, t \sigma)$ from (1). If we put

$$
B(u)=\sum_{i=1}^{r} A\left(u, t_{i}\right)
$$

for every $u \in G$, then $B(u)$ is determined uniquely irrespective of the choice of the representatives $t_{i}$ in the cosets $t_{i} S$. Then we have from (1)

$$
B(u)-B(u v)+A(u) B(v)=r A(u, v)
$$

for every $u, v \in G$. Since the index $r$ of $S$ is prime to $p, A(u, v)$ splits relative to $G$.

By this theorem we see that the extension $\bar{G}$ is completely determined by the representation $\Lambda$ and the part of the factor set for a $p$-Sylow subgroup $S$. When in particular the order of $G$ is prime to $p$, then $\bar{G}$ is determined completely by $A$. Next we consider the case, where $A$ is irreducible. This means that the 
$G$-module $H$ is irreducible, i.e. $H$ has no proper subgroup, which is an invariant subgroup of $\bar{G}$. In this case the extension $\bar{G}$ is called irreducible. When $\bar{G}$ is not irreducible, it can be obtained by repeating irreducible extensions. In fact, choose an irreducible $G$-submodule $H_{1}$ of $H$. Then $\bar{G}$ becomes an irreducible extension of. $H_{1}$ by $\bar{G} / H_{1}$ and $\bar{G} / H_{1}$ an extension of $H / H_{1}$ by $G$, and so forth. Now let $\bar{S}$ be the subgroup of $\bar{G}$, which corresponds to $S$ in the natural homomorphism $\bar{G} \rightarrow G$. By a theorem on finite groups it follows that the intersection of $H$ and the center of $\bar{S}$ has a vector, which is different from zero, since $\bar{S}$ is a $p$-group. Consequently there exists $h \neq 0$ in $H$, such that $\sigma h=h$ for every $\sigma \in S$. The submodule of $H$, which is generated by $t_{i} h, i=1, \ldots, r$, is a $G$ module and hence is identified with $H$, since $H$ is an irreducible $G$-module. Then we can assume that $t_{1} h, \ldots, t_{n} h$ form a basis of $H$, where $n \leqq r$. If in particular $S$ is invariant, then $H$ becomes a $G / S$-module and yields an irreducible representation $A$ of the factor group $G / S$.

Every element $u \in G$ induces a permutation of all left cosets $t_{i} S$ with $u t_{i} S$ $=t_{i(u)} S$ and hence a permutation $i \rightarrow i(u)$ of indices $i$ with $i(u v)=i(v)(u)$. Let the matrix $A_{0}(u)=\left(\lambda_{i, j}(u)\right)$ be determined such that $\lambda_{i j}(u)=1$, if $i=j(u)$, and $\lambda_{i j}(u)=0$, if $i \neq j(u)$. Then $A_{0}(u)$ yields a representation $A_{0}$ of $G$, which is induced by the identical representation of $S$. If in particular $S$ is invariant, then $A_{0}$ is the regular representation of the factor group $G / S$. We can assume that $t_{1}$ is the identity of $G$ and, putting

$$
h_{1}=\left(\begin{array}{c}
1 \\
0 \\
\vdots \\
0
\end{array}\right),
$$

we have

$$
h_{i}=t_{i} h_{1}=A_{9}\left(t_{i}\right) h_{1}=\left(\begin{array}{c}
0 \\
\vdots \\
1 \\
\vdots \\
0
\end{array}\right) .
$$

We denote with $H_{0}$ the $G$-module, which is gererated by $h_{i}, i=1, \ldots, r$. An extension $\bar{G}_{0}$ of $H_{0}$ by $G$ with the representation $A_{0}$ shall be called regular. The following theorem asserts that every irreducible extension can be obtained by means of a certain regular extension, if $S$ is invariant.

THEOREM 3. Let $\bar{G}$ be an irreducible extension of $H$ by $G$. If the p-Sylow subgroup $S$ of $G$ is invariant, then there exists a regular extension $\bar{G}_{0}$ of $H_{0}$ by $G$ and a submodule $\bar{H}$ of $H_{0}$, such that $\bar{G}$ is isomorphic with $\bar{G}_{0} / \bar{H}$ and $H$ corresponds to $H_{0} / H$.

Proof. Since the order of $G / S$ is prime to $p$, its regular representation $1_{0}$ 
is completely reducible. There exists a submodule $H_{1}$ of $H_{0}$ with $H_{0}=H_{1}+H_{2}$, such that $H$ is operator-isomorphic with $H_{1}$. If $A$ is the irreducible representation of $G$ by $H$, then we have

$$
D A_{0} D^{-1}=\left(\begin{array}{cc}
A & 0 \\
0 & X
\end{array}\right) .
$$

Let $A(s, t)$ be the factor set of the extension $\bar{G}$. Putting

$$
A(s, t)=\left(\begin{array}{c}
a_{1}(s, t) \\
\vdots \\
a_{n}(s, t)
\end{array}\right),
$$

we consider the $r$-dimensional vector

$$
\bar{A}(s, t)=\left(\begin{array}{c}
a_{1}(s, t) \\
\vdots \\
a_{n}(s, t) \\
0 \\
\vdots \\
0
\end{array}\right) .
$$

Then this becomes a factor set for the representation $D \Lambda_{0} D^{-1}$ and yields a regular extension $\bar{G}_{0}$ of $H_{0} b y G$. The factor group $\bar{G}_{0} / H_{2}$ is now an extension of $H_{0} / H_{2}$ by $G$, where $H_{0} / H_{2}$ is isomorphic with $H$. Its factor set can be identified with $A(s, t)$, the representation being $A$. Hence $\bar{G}$ is isomorphic with $\bar{G}_{0} / H_{2}$ and $H$ corresponds to $H_{0} / H_{2}$.

If $S$ is invariant, then $\Lambda(\sigma)$ is the unit matrix for every $\sigma \in S$. Hence every component $a(\sigma, \tau)$ of the factor set $A(\sigma, \tau)$ for an irreducible extension satisfies the relation

$$
a(\sigma, \tau)+a(\sigma \tau, \varphi)=a(\sigma, \tau \varphi)+a(\tau, \varphi)
$$

for $\sigma, \tau, \varphi \in S$. This is also satisfied by every component of the factor set for a regular extension, since $\Lambda_{0}(\sigma)$ is the unit matrix for $\sigma \in S$. From the preceding lemma we have

$$
\Lambda_{0}\left(t_{i}\right) A\left(t_{i}^{-1} \sigma t_{i}, t_{i}^{-1} \tau t_{i}\right)=A(\sigma, \tau)+B(\sigma, i)-B(\sigma \tau, i)+\Lambda_{0}(\sigma) B(\tau, i) .
$$

If we consider only the $i$-th components, then this implies

$$
a_{1}\left(t_{i}^{-1} \sigma t_{i}, t_{i}^{-1} \tau t_{i}\right)=a_{i}(\sigma, \tau)+b_{i}(\sigma, i)-b_{i}(\sigma \tau, i)+b_{i}(\tau, i) .
$$

Now, putting 


$$
A^{\prime}(\sigma, \tau)=\left(\begin{array}{c}
a_{1}\left(t_{1}^{-1} \sigma t_{1}, t_{1}^{-1} \tau t_{1}\right) \\
\vdots \\
a_{1}\left(t_{r}^{-1} \sigma t_{r}, t_{r}^{-1} \tau t_{r}\right)
\end{array}\right), \quad B^{\prime}(\sigma)=\left(\begin{array}{c}
b_{1}(\sigma, 1) \\
\vdots \\
b_{r}(\sigma, r)
\end{array}\right),
$$

we have

$$
A^{\prime}(\sigma, \tau)=A(\sigma, \tau)+B^{\prime}(\sigma)-B^{\prime}(\sigma \tau)+\Lambda_{0}(\sigma) B^{\prime}(\tau)
$$

for $\sigma, \tau \in S$. If we choose $B^{\prime}(s)$ arbitrarily, when $s$ does not belong to $S$, then we can extend $A^{\prime}(\sigma, \tau)$ to a factor set $A^{\prime}(s, t)$, which is equivalent to $A(s, t)$ by theorem 2 , such that

$$
A^{\prime}(s, t)=A(s, t)+B^{\prime}(s)-B^{\prime}(s t)+A_{0}(s) B^{\prime}(t) .
$$

The vectors $A^{\prime}(\sigma, \tau)$ can be determined only by the values of the first components $a_{1}(\sigma, \tau)$ of $A(\sigma, \tau)$ for all $\sigma, \tau \in S$. The set of values $a_{1}(\sigma, \tau)$ is called the fundamental component of the factor set for the regular extension and denoted with $a(\sigma, \tau)$ in place of $a_{1}(\sigma, \tau)$. We say that two fundamental components $a(\sigma, \tau)$ and $a^{\prime}(\sigma, \tau)$ are equivalent, if there exist integers $b(\sigma)$ mod. $p$ such that

$$
a^{\prime}(\sigma, \tau)=a(\sigma, \tau)+b(\sigma)-b(\sigma \tau)+b(\tau)
$$

for all $\sigma, \tau \in S$. Two fundamental components yield a same regular extension up to isomorphism, if and only if they are equivalent. We suppose that it holds

$$
D A_{0} D^{-1}=\left(\begin{array}{cccc}
A_{1} & 0 & \cdots & 0 \\
0 & A_{2} & \cdots & 0 \\
\vdots & \vdots & \ddots & \vdots \\
\vdots & \vdots & \ddots & \vdots \\
0 & 0 & \cdots & A_{m}
\end{array}\right)
$$

where $A_{i}$ are irreducible. Then the factor set $D A^{\prime}(\sigma, \tau)$ decomposes into $A_{i}(\sigma, \tau)$, $i=1, \ldots, m$, where $A_{i}(\sigma, \tau)$ is referred to $A_{i}$ respectively. We observe that the fundamental component $a(\sigma, \tau)$ of a regular extension is a linear combination of components of factor sets of all irreducible extensions, which can be obtained from the regular extension. Conversely every such irreducible extension is completely determined by $\Lambda_{i}$ and $a(\sigma, \tau)$. We say that each irreducible extension, which can be obtained by $a(\sigma, \tau)$, is referred to $a(\sigma, \tau)$.

We shall now pass to the imbedding of a normal extension $K$ over $k$, whose Galois group is $G$. Let $\Omega$ be the subfield of $K$, which corresponds to the $p$-Sylow subgroup $S$ of $G$. We assume that $k$ contains a primitive $p$-th root $\zeta$ of unity. If $a(\sigma, \tau)$ is a fundamental component of the factor set for a regular extension, then the $a(\sigma, \tau)$-th powers of $\zeta$ become a factor set with respect to $S$ and $K$ by virtue of (2). If $a(\sigma, \tau)$ and $\boldsymbol{a}^{\prime}(\sigma, \tau)$ are equivalent, then they yield associated factor sets with respect to $S$ and $K$. If there exists $\xi_{\sigma} \in K$ such that the $a(\sigma$, $\tau)$-th power of $\zeta$ is equal to $\sigma\left(\xi_{\tau}\right) \xi_{\sigma \tau}^{-1} \xi_{0}$ for all $\sigma, \tau$ from $S$, then we say that it splits. 
THEOREM 4. Suppose that $k$ contains a primitive $p$-th root $\zeta$ of unity and the $p$-Sylow subgroup $S$ of $G$ is invariant. The necessary and sufficient condition, under which the imbedding of $K$ for every irreducible extension by $G$ referred to a fundamental component $a(\sigma, \tau)$ is possible, is that the factor set $\zeta^{a(\sigma, \tau)}$ with respect to $S$ and $K$ splits.

First we shall prove that the condition is necessary. Let $\bar{G}$ be an irreducible extension of $H$ by $G$ with the fundamental component $a(\sigma, \tau)$ and the Galois group of $L$ over $k$ be $\bar{G}$, where $K$ corresponds to $H$. We choose $h \in H$ such that $t_{1} h, \ldots, t_{n} h$ constitute a basis of $H$, where $\sigma h=h$ for all $\sigma \in S$. To the subgroup $H_{i}$ of $H$, which is generated by all elements of the basis except $t_{i} h$, corresponds a subfield $L_{i}=K\left(\sqrt[t]{\alpha_{i}}\right)$ of $L$ with $\alpha_{i} \in K$. We can assume that $t_{i} h$ induces the automorphism of $L_{i}$ with $\sqrt[D]{\alpha_{i}} \rightarrow \zeta \sqrt{\alpha_{i}}$. An automorphism $g_{o}$ of $L$ over $k$ induces $\beta \rightarrow \sigma(\beta)$ for $\beta \in K$. Since $H_{i}$ is an invariant subgroup of $\bar{S}$, the field $L_{i}$ is normal over $\Omega$. Hence we have $g_{\sigma}\left(\sqrt[D]{\alpha_{i}}\right)=\sqrt[D]{\alpha_{i}} \xi_{\sigma}$ with $\xi_{\sigma} \in K$. Now let $g_{\sigma} g_{\tau}=A(\sigma, \tau) g_{\sigma}$ with $A(\sigma, \tau) \in H$ and $a_{i}(\sigma, \tau)$ be the $i$-th component of $A(\sigma, \tau)$. Then the automorphism $A(\sigma, \tau)$ induces

$$
\sqrt[p]{\alpha_{i}} \rightarrow \zeta^{a_{i}(\sigma, \tau)} \sqrt[p]{\alpha_{i}}
$$

It follows then from $g_{s} g_{\tau}\left(\sqrt[p]{\alpha_{i}}\right)=A(\sigma, \tau) g_{\sigma \tau}\left(\sqrt[D]{\alpha_{i}}\right)$ the relation

$$
\sqrt[p]{\alpha_{i}} \xi_{\sigma} \cdot \sigma\left(\xi_{\tau}\right)=\zeta^{a_{i}(\sigma, \tau)} \sqrt[p]{\alpha_{i} \xi_{\sigma \tau}} .
$$

Hence the $a_{i}(\sigma, \tau)$-th power of $\zeta$ splits. Since $a(\sigma, \tau)$ is a linear combination of all components $a_{i}(\sigma, \tau)$ for all irreducible extensions, which are referred to $a(\sigma, \tau)$, we can readily see that the $a(\sigma, \tau)$-th power of $\zeta$ splits.

Next we prove that the condition is sufficient. By Speiser's theorem we have $\xi_{\sigma}^{p}=\alpha^{g-1}$ with $\alpha \in K$ for all $\sigma \in S$. We choose a prime ideal $q$ in $\Omega$ with degree one, such that $q$ is prime to all conjugates of $\alpha$ and does not ramify in $K$. Choose a number $c$ in $Q$ under following conditions: (1) $c$ is divisible by $q$ and not divisible by the square of $q,(2) c$ is prime to all conjugate prime ideals of $q$ except $q$. Putting $\alpha c=\beta$ we have $\beta^{\sigma-1}=\xi_{\sigma}^{p}$. We put $\beta_{i}=t_{i}(\beta)$ and $\gamma=\Pi \beta_{i}^{i}$, where $c_{i}$ are rational integers. Then $\gamma$ becomes a $p$-th power of a number in $K$, if and only if all $c_{i}$ are divisible by $p$. Now let $L$ be a field generated over $K$ by adjoining ali numbers $\sqrt[D]{\beta_{i}}, i=1, \ldots, r$. The extension $L$ is normal over $k$ and abelian over $K$ with the Galois group $H_{0}$, which is abelian of type $(p, \ldots, p)$ and of rank $r . H_{0}$ has a basis $h_{1}, \ldots, h_{r}$, where $h_{i}$ induces

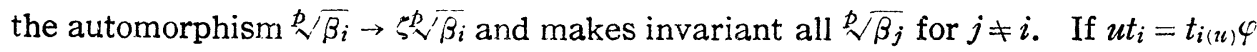
for $u \in G$ with $\varphi \in S$. we choose the automorphism $g_{u}$ of $L / k$ with

$$
\sqrt[D]{\beta_{i}} \rightarrow t_{i(u)}\left(\xi_{\varphi}\right) \stackrel{D^{\prime}}{\beta_{i(u)}} \text {. }
$$

Then we can readily see that it holds $g_{u} h_{i} g_{u}^{-1}=h_{i, u}$ ) and hence $H_{0}$ yields the 
representation $1_{0}$. Also it is easily verified that we obtain $g_{\sigma} g_{\tau}=A(\sigma, \tau) g_{\sigma \tau}$, where $A(\sigma, \tau)$ is a product of $a\left(t_{i}^{-1} \sigma t_{i}, t_{i}^{-1} \tau t_{i}\right)$-th powers of $h_{i}, i=1, \ldots, r$. Therefore the Galois group of $L$ over $k$ is the regular extension of $H_{n}$ by $G$ with the fundamental component $a(\sigma, \tau)$. The imbedding is now possible for every irreducible extension referred to $a(\sigma, \tau)$ by Galois theory and theorem 3 .

COROLlaRy. If the order of $G$ is prime to $p$ and $k$ contains a primitive $p$-th root $\zeta$ of unity, then the imbedding of $K$ is possible for every irreducible extension of $H$ by $G$.

The case, where a $p$-Sylow subgroup of $G$ is not invariant, is rather complicated and seems difficult to obtain a simple condition, under which the imbedding is possible.

\section{REFERENCES}

[1] R. Brauer, Über die Konstruktion der Schiefkörper, die von endlichem Rang in bezug auf ein gegebenes Zentrum sind. J. reine angew. Math. 168 (1932).

[2] H. Reichardt, Konstruktion von Zahlkörpern mit gegebener Galoisgruppe von Primzahlpotenzordnung. J. reine angew. Math. 177 (1937).

[3] H. Richter, Über die Lösbarkeit einiger nicht-abelscher Eintettungsprobleme, Math. Ann. 112 (1936).

[4] A. Scholz, Über die Bildung algebraischer Zahlkörper mit aufösbarer Galoisscher Gruppe, Math. Z. 30 (1929).

[5] A. Scholz, Reduktion der Konstruktion von Körpern mit zweistufiger metabelscher Gruppe, Heidelberger Akad. Sitzungsber. (1929).

[6] A. Scholz, Konstruktion algebraischer Zahlkörper beliebiger Gruppe von Primzahlpotenzordnung I, Math. Z. 42 (1937).

[7] T. Tannaka, Über die Konstruktion der galoischen Körper mit vorgegebener $p$-Gruppe, Tôhoku Math. J. 43 (1937).

[8] H. Zassenhaus, Lehrbuch der Gruppentheorie.

Mathematical Institute, Ochanomizu University 